\title{
Assessment of Serum ST2 Receptor Levels in Female Coronary Artery Disease Patients with LV Dysfunction in Comparison to Males
}

\author{
Y. Rama Kishore ${ }^{1} \quad$ N. Lalitha ${ }^{1} \quad$ M. Naveen Kumar ${ }^{1}$ \\ ${ }^{1}$ Department of Cardiology, NIMS, Hyderabad, India
}

Ind J Car Dis Wom:2020;5:88-94

\begin{abstract}
Address for correspondence Rama Kishore Yalampati, DM, Department of Cardiology, NIMS, Hyderabad, TS- 500 082, India (e-mail: honeyyrk@gmail.com).
\end{abstract}

\begin{abstract}
Keywords

- ST2

- LV dysfunction

- coronary artery disease

Background Heart failure is shown to be associated with elevated levels of ST2 levels in blood. The data about the relation of blood ST2 levels, in patients of significant coronary artery disease (CAD) with left ventricular (LV) dysfunction is limited. The impact of gender on ST2 levels not studied until now, so this study aimed to assess the blood ST2 levels measured for males and females patients in comparisons, of CAD with LV dysfunction and their relation to the outcomes at the end of 1-year post intervention. Material and Methods Out of the 60 patients with 1:2 ratio of females and males with CAD and LV dysfunction, baseline quantitative assessment of ST2 levels in blood was done by rapid lateral flow immunoassay method; $>35 \mathrm{mg} / \mathrm{mL}$ was considered as abnormal. Postintervention patients followed up to one year, adverse cardiovascular events noted at the end of 1 year. By using binary logistic regression, the outcomes in females compared with males for statistical significance in relation to baseline blood ST 2 levels. The $p$ value of $<0.05$ considered was significant.

Results Among the 60 patients (females $33.3 \%$ and males $66.6 \%$ ) presented with CAD with LV dysfunction in the study, 40 (66.6 \%) patients diagnosed of acute coronary syndrome (ACS), and 20 (33.3\%) patients with chronic stable angina (CSA). In both the genders, ACS is most common presentation (55\% in females and $72.5 \%$ in males). Eighteen (90\%) females and $20(50 \%)$ males were hypertensive, 12 (60\%) females and 18 (45\%) males were diabetics. Twenty-seven (67.5\%) males were smokers and 14 (35\%) were alcoholics. Nine (45\%) female and 14 (35\%) male patients had elevated ST2 levels. At the end of 1 year 9 females had events including 2 mortalities, 2 heart failures, and 7 repeat revascularizations. At the same time 7 male patients had events at the end of 1 year including 4 mortalities, 5 heart failures, and 2 repeat revascularizations. The difference in the event rates between male and female patients were statistically significance $(p=0.03)$. Elevated ST2 levels were correlated with echocardiographic parameter end systolic volume which was statistically significant $(p=0.03)$. Similarly elevated ST2 levels correlated with presence of diabetes $(p=0.01)$ and low LV EF.

Conclusion Mean Blood ST2 levels were higher in females who associated with diabetes, high echo cardio graphic end-systolic volume, and a low ejection fraction of LV in severe LV dysfunction with statistical significance. At the end of 1 year in patients with elevated baseline ST2 levels, the female patient had more events than males with statistical significance.
\end{abstract}




\section{Abstract Image}

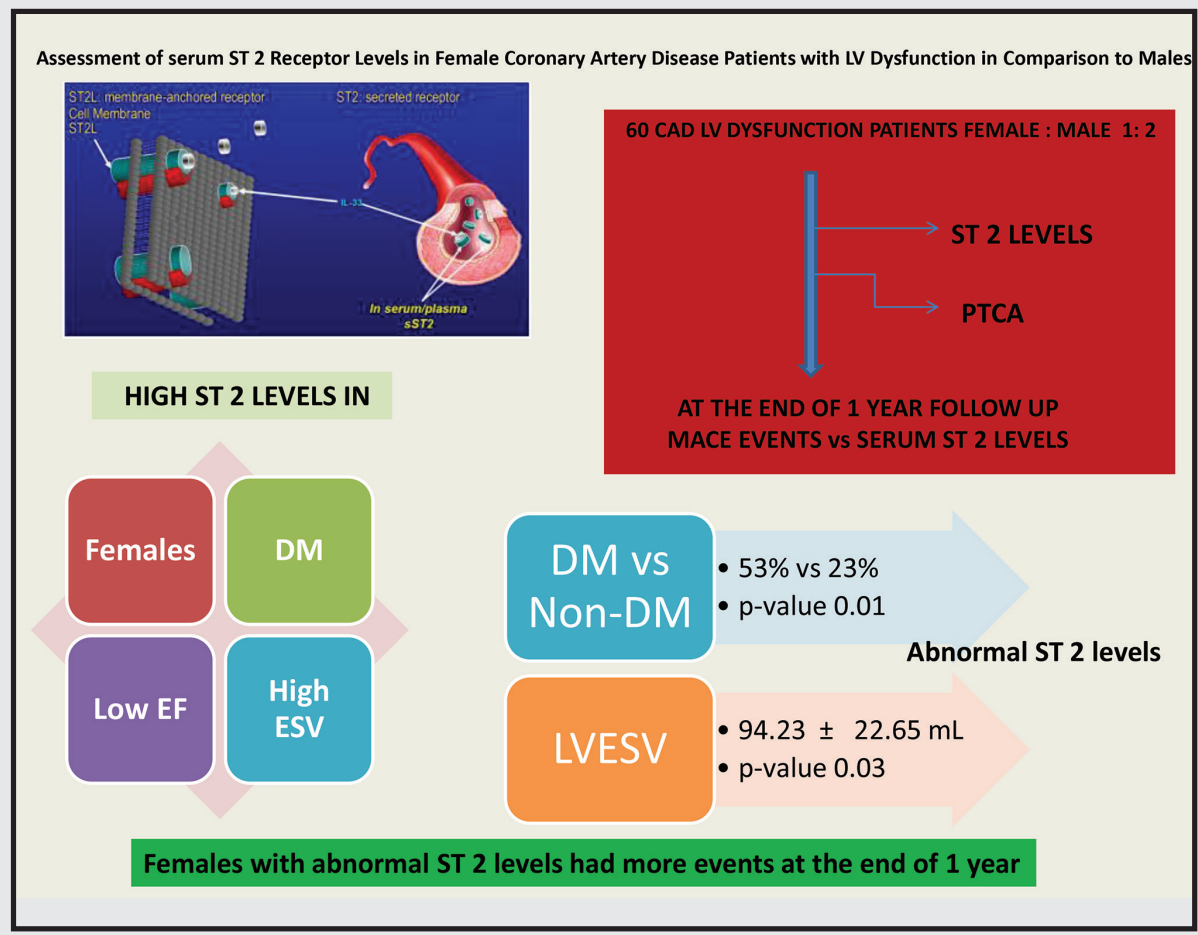

\section{Introduction}

Heart failure (HF), a clinical entity characterized by insult to the myocardium, neurohormonal activation, remodeling, involves activation of multiple signaling pathways. With each subsequent HF hospitalization, there is an increase in mortality risk and a reduction in the quality of life. ${ }^{1}$ Assessment of different molecules participating in various steps of these pathways established their role as biomarkers. Among them, troponin and natriuretic peptides were already included in the heart failure guidelines, which influenced water and salt handling as well as pressure regulation, resulting in the alteration of function and structure of the myocardium.

Soluble ST2 (sST2) is a new biomarker in the evaluation and management of heart failure. ST2 belongs to interleukin-1 receptor family and stands for suppression of tumorigenicity 2 and is also known as interleukin 1 receptor like 1 (IL1RL-1).,3

ST2 has two isoforms: transmembrane or cellular (ST2L) and soluble or circulating (sST2) forms. It is released by all living cells in response to cell damage. In response to cardiac injury, the ST2 system, which is cardio protective, is upregulated in myocytes and fibroblasts. ${ }^{4}$ After myocardial infarction (MI), it reduces the extent of cardiac damage by reducing cardio myocytes' apoptosis, inflammatory cardiac activation, fibrosis and adverse myocardial remodelling. Soluble ST2 (sST2) acts as a decoy receptor and prevents the beneficial effects of IL-33/ST2L interaction. ${ }^{5}$ Soluble ST2 (sST2) is a powerful independent prognostic biomarker in acute MI, and acute decompensated and chronic heart failure.

Blood ST2 levels reflect ventricular wall stress, inflammation and macrophage activation. In an acute MI, elevated
ST2 levels of blood can be utilized for risk stratification to recognize patients at high-risk of developing adverse cardiovascular events like heart failure or death. The current ACC/ AHA guidelines recommend monitoring of blood ST2 levels in heart failure patients for risk stratification, as a class II recommendation with level of evidence B. ${ }^{6}$

Expression of ST2 is related to stretch mechanism in the myocardium. Although in vitro studies suggested secretion of sST2 from myofibroblasts via mechanoreceptors, in vivo release of sST2 is in response to an inflammatory process. We assumed that, similar to other markers of inflammation (tumor necrosis factor, hsCRP, or IL-6), increased sST2 may signal the severity of cardiomyopathy; also, there is limited data about the ST2 levels in different degrees of left ventricular (LV) dysfunction in patients with significant obstructive coronary artery disease (CAD). Till now, there are no studies that assessed the ST2 levels in patients of CAD with LV dysfunction.

Although it has been shown that ST2 concentrations correlate with heart failure severity, there is no definite quantitative level that perfectly separates patients with and without heart failure for disease diagnosis; 7 also, it was proven that plasma BNP values increase with age and are higher in women than men. ${ }^{8}$ Similarly, there are no studies that had observed the role of ST2 in relation to cardiovascular outcomes comparing males with females.

\section{Aim of the Study}

This study was aimed to assess the role of blood ST2 levels at the time of presentation in female patients of CAD with LV 
dysfunction and their relation to the outcomes at the end of 1 year after intervention and compare the same with male patients.

\section{Materials and Methods}

The study was conducted from May 2018 after ethics committee approval from the institute. After obtaining the written and informed consent, 60 participants of CAD with LV dysfunction were enrolled into the study with female to male ratio of 1:2 (i.e., 20 female patients and 40 male patients); base line blood ST2 levels were estimated and then treated as per protocol.

\section{ST2 Estimation}

The quantitative determination of ST2 in venous ethylenediamine tetra-acetic acid (EDTA) anticoagulated serum is carried out by the ASPECT-PLUS ST2 Test (Critical Diagnostics Limited, 23 Mespil Road, Dublin 4, and Ireland), which is based on rapid lateral flow immunoassay and is to be used with the ASPECT Reader TM.

This test is a quantitative sandwich monoclonal lateral flow immunoassay; the sample well was loaded with EDTA sample where it flows through the strip containing anti-ST2 antibodies.

The second well contains assay buffer. The cassette is then inserted into the ASPECT Reader for incubation, and ST2 is quantitatively estimated and displayed by the reader. Quantitative estimation of ST2 value using this test is produced by linear regression analysis and a linear calibration curve unique to each lot of ASPECT-PLUS ST2 Test, which is coded on each test cassette. The fluorescent signal measured on the test line is evaluated against the calibration curve for the calculation of ST2 concentration. A value $\leq 35 \mathrm{ng} / \mathrm{mL}$ was considered normal. The test could detect values as low as $12.5 \mathrm{ng} / \mathrm{mL}$ and as high as $250 \mathrm{ng} / \mathrm{mL}^{9}$

After enrolling the patient in the study, patients underwent a detailed 2D echo study; patients with acute coronary syndrome underwent percutaneous coronary intervention after the coronary angiogram. In patients with LV dysfunction and chronic stable angina (CSA) symptoms, after the coronary angiogram, percutaneous transluminal coronary angioplasty (PTCA) was done in patients with significant viable myocardium in the MRI viability scan.

Patients were followed-up to one year, and adverse cardiovascular events were noted at the end of 1 year. The outcomes in females were compared with that of males for statistical significance in relation to baseline blood ST 2 levels.

\section{Statistical Analysis}

All the collected data were represented as the mean \pm standard deviation (SD), and comparisons were done by using the binary logistic regression. Minitab version 17 by Minitab, Ltd, United Kingdom was used to perform statistical analysis. The $p$ value of $<0.05$ was considered as statistically significant. Chi-square/Fisher's exact test was used to compare the statistical significance of categorical variables.

\section{Results}

When the type of presentation was compared between males and females, in females, 11 (55\%) patients were afflicted with acute coronary syndrome (ACS), 9 (45\%) patients had CSA, $9(45 \%)$ patients had moderate left ventricular (LV) dysfunction, 11 (55\%) patients had severe LV Dysfunction (-Fig. 1). While among males, 29 (72.5\%) patients were afflicted with ACS, 11 (27.5\%) patients had CSA, 17 (42.5\%) patients had moderate LV dysfunction, and 23 (57.5\%) patients had severe LV dysfunction.

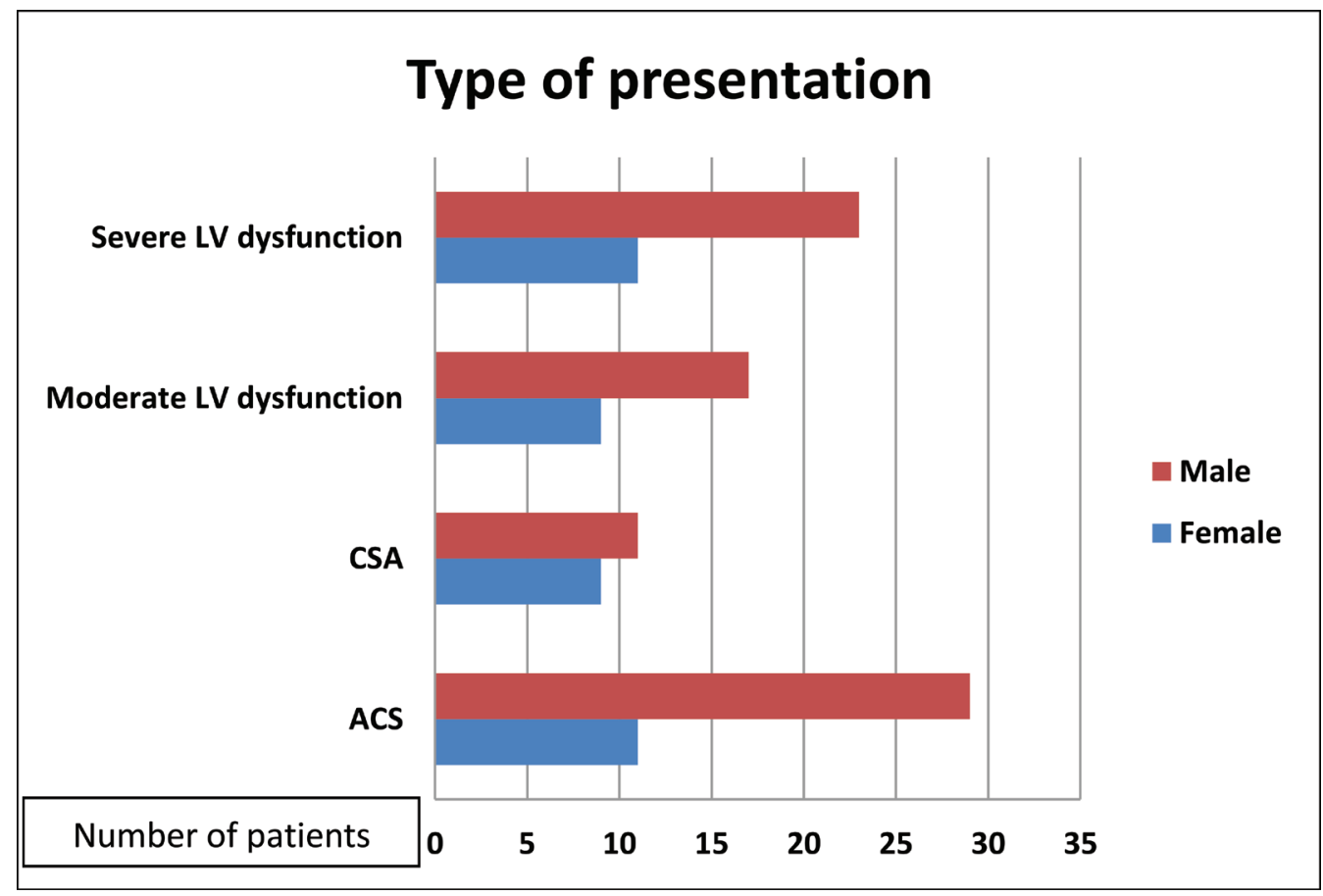

Fig. 1 Type of presentation in females versus males. 
When risk factors were compared between males and females, in females, 18 (90\%) were hypertensivs, 12 (60\%) were diabetics, and $1(5 \%)$ patient was a smoker and alcoholic. While in males, 20 (50\%) were hypertensive,18 (45\%) were diabetic, 27 (67.5\%) patients were smokers, and 14 (35\%) were alcoholics.

Although all the study patients had LV dysfunction, ST 2 levels were normal in 61 (47/60) percent of the patients, and abnormal ST2 levels were seen in 39\% (23/60) of the patients. With 14 patients (35\%), males were the predominant population with abnormal ST 2 levels than female patients 9 (45\%).

The baseline mean ST 2 levels in females was $61.9 \pm 71.6 \mathrm{ng} / \mathrm{mL}$ and $37.0 \pm 37.9 \mathrm{ng} / \mathrm{mL}$ among males; the difference did not show statistical significance.

In patients with major adverse critical events (MACE) at the end of one year, $45 \%$ of females were with elevated ST2 levels and $17.5 \%$ of males were with abnormal ST 2 levels and the difference showed statistical significance when the risk factors, echo parameters, and angiographic parameters were correlated with difference between abnormal ST 2 levels and normal ST 2 levels. Only diabetes had significant correlation ( $p$ value of 0.01 ), while gender, hypertension (HTN), smoking, alcoholism, type of presentation, degree of LV dysfunction, echo parameters, and angiographic profile did not show any statistical significance $(p>0.05)$ when echocardiographic parameters of end diastolic volume (EDV), end systolic volume (ESV), end diastolic diameter (EDD), end systolic diameter (ESD), ejection fraction (EF), deceleration time (DT), and E/e' ratio were correlated with abnormal mean ST2 levels of study population. Only ESV showed statistical significance with a $p$ value of $0.03(<0.05)$, while other parameters did not show statistical significance, that is, $p>0.05$.

\section{Discussion}

Myocardial injury is followed by cardiac remodeling by the activation of multiple neurohumoral mechanisms, which ultimately lead to the formation of cardiac fibrosis. Understanding these pathways has become the basis for the measurement of biomarkers to diagnose and monitor treatment, and also multiple pharmacological options are targeted to prevent the disease progression. ${ }^{10}$

There are several biomarkers for the diagnosis, management and prognostication in patients with cardiovascular disease. A potential mediator of cardiac fibrosis that was found in the recent times is the interleukin-33 (IL-33)/suppression of tumorigenicity 2 (ST2) pathway, which belongs to the IL-1 family of cytokines. Soluble ST2 (sST2) is one of the main isoforms of ST2 with strong prognostic value in cardiovascular disease. ${ }^{11}$

ST2 is a marker of myocardial fibrosis and remodelling and holds promising as a biomarker of prognostication in acute $\mathrm{MI}$ and heart failure. Increased serum concentrations of sST2 observed after MI are associated with increased risk of mortality and development of heart failure (HF). ${ }^{12,13}$
Weinberg et al were the first to show that sST2 levels increased in patients after myocardial infarction. The increase correlated with peak creatine kinase positively and negatively with left ventricle ejection. Weir et al reported sST2 is related to the size of the infarct and its evolution over 24 weeks in patients after acute MI and resultant LV dysfunction. ${ }^{13}$

We assessed the blood ST2 levels at the time of presentation in female patients with CAD and LV dysfunction and their relation to the outcomes at the end of 1 year after intervention and compared the same with that of male patients.

In our study, among 60 patients recruited in the study, there were 20 females (33.3\%) and 40 males (66.6\%). The mean age of presentation, irrespective of gender, was $59.02 \pm 9.62$ years. The mean age of presentation was 3.6 years lesser for men compared with women (56.29 versus 59.83 years). This was comparable to the previous studies in India. In a study by Kunwar et al, which assessed the reperfusion trends in 1459 STEMI patients, the mean age of the presentation was $56.3 \pm 11.8$ years, and in ACS patients of CREATE registry, it was $57.5 \pm 12$ years. ${ }^{14,15}$

At the time of presentation, 40 (66.6\%) patients had ACS and 20 (33.3\%) patients had CSA (-Fig. 1). Among 20 female patients, 11 (55\%) patients had ACS and $9(45 \%)$ patients were CSA. In males, 29 (72.5\%) patients had ACS and 11 (27.5\%) patients had CSA, so in our study, ACS was the most common presentation of CAD in both the genders.

Values for sST2 differ among men and women, increase with age, and were associated with diabetes and hypertension in the community-based Framingham Heart Study. ${ }^{16}$ However, from the results of the Dallas study, we concluded that sST2 did not correlate with CAD risk factors such as dyslipidemia, HTN, smoking, and common carotid intima-media thickness. ${ }^{17}$

In our study, when risk factors (-Fig. 2 and - Table 3) were analyzed, overall HTN is the most common risk factor (63.3\%) followed by diabetes mellitus (DM) Type II (50\%). In females, 18 (90\%) were hypertensive, 12 (60\%) were diabetic, and 1 (5\%) patient was a smoker and alcoholic; also, among them, 9 (45\%) patients had moderate LV dysfunction and 11 (55\%) patients had severe LV dysfunction. While in males, 20 (50\%) were hypertensive, 18 (45\%) were diabetic, 27 (67.5\%) patients were smokers, and 14(35\%) were alcoholics; also, among them, 17 (42.5\%) patients had moderate LV dysfunction and 23 (57.5\%) patients had severe LV dysfunction. So, the most common risk factor in females was HTN, followed by diabetes. In males, the most common risk factor was smoking, followed by diabetes and HTN. Severe LV dysfunction was most common in females. These observations were similar to what was found in the INTERHEART Study. Only DM has shown significant correlation with abnormal ST2 levels with statistical significance.

At the time of presentation (-Fig. 3), among 20 females, 9 (45\%) patients has elevated ST 2 levels, that is more than $12.5 \mathrm{ng} / \mathrm{mL}$, while 11 (55\%) patients had shown normal ST2 levels. Fourteen (35\%) patients had elevated levels in males, with normal ST2 levels in 26 patients (65\%), so the ST2 levels were found to be higher in females than males, although the 


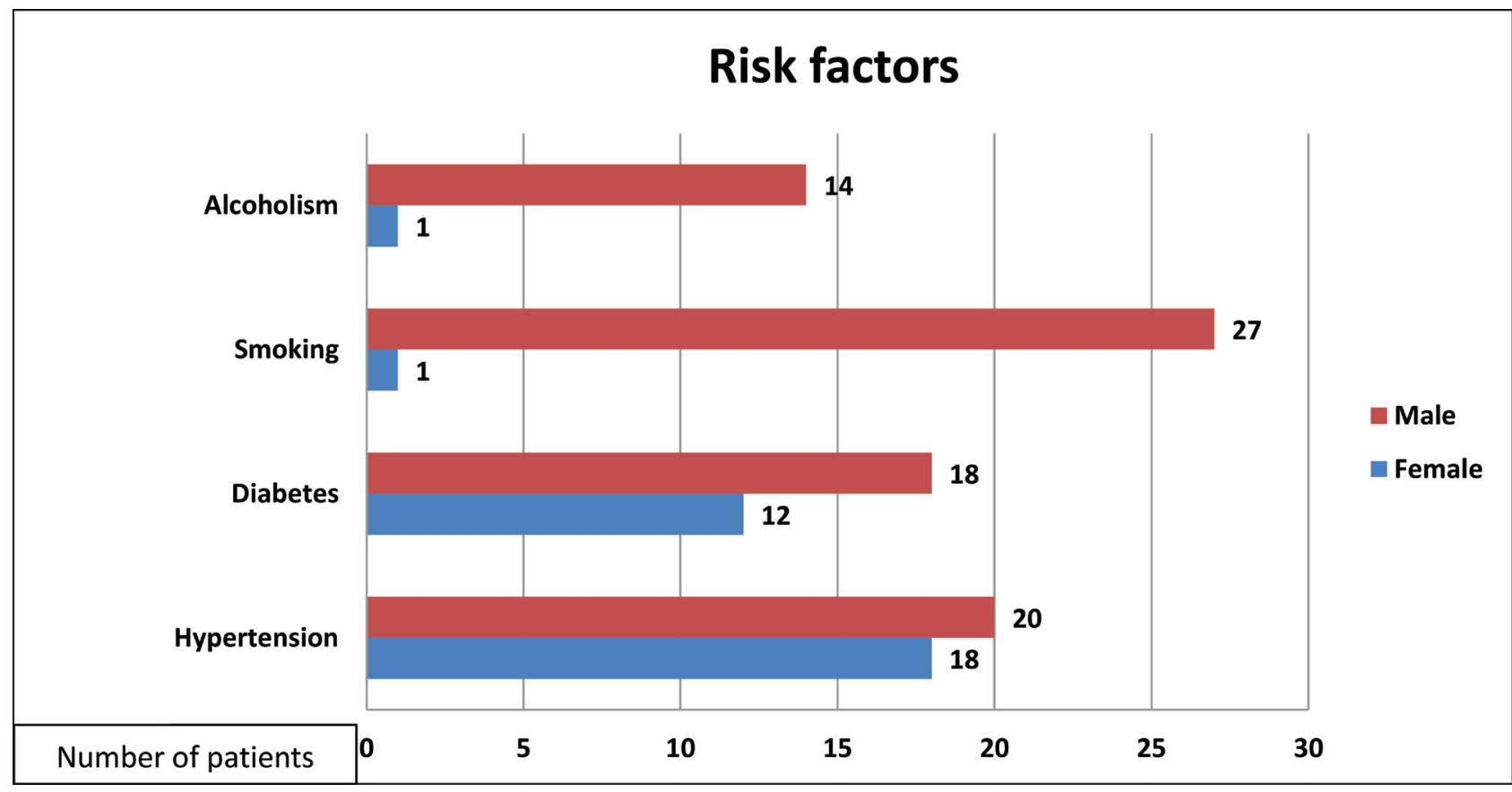

Fig. 2 Risk factors in females versus males.

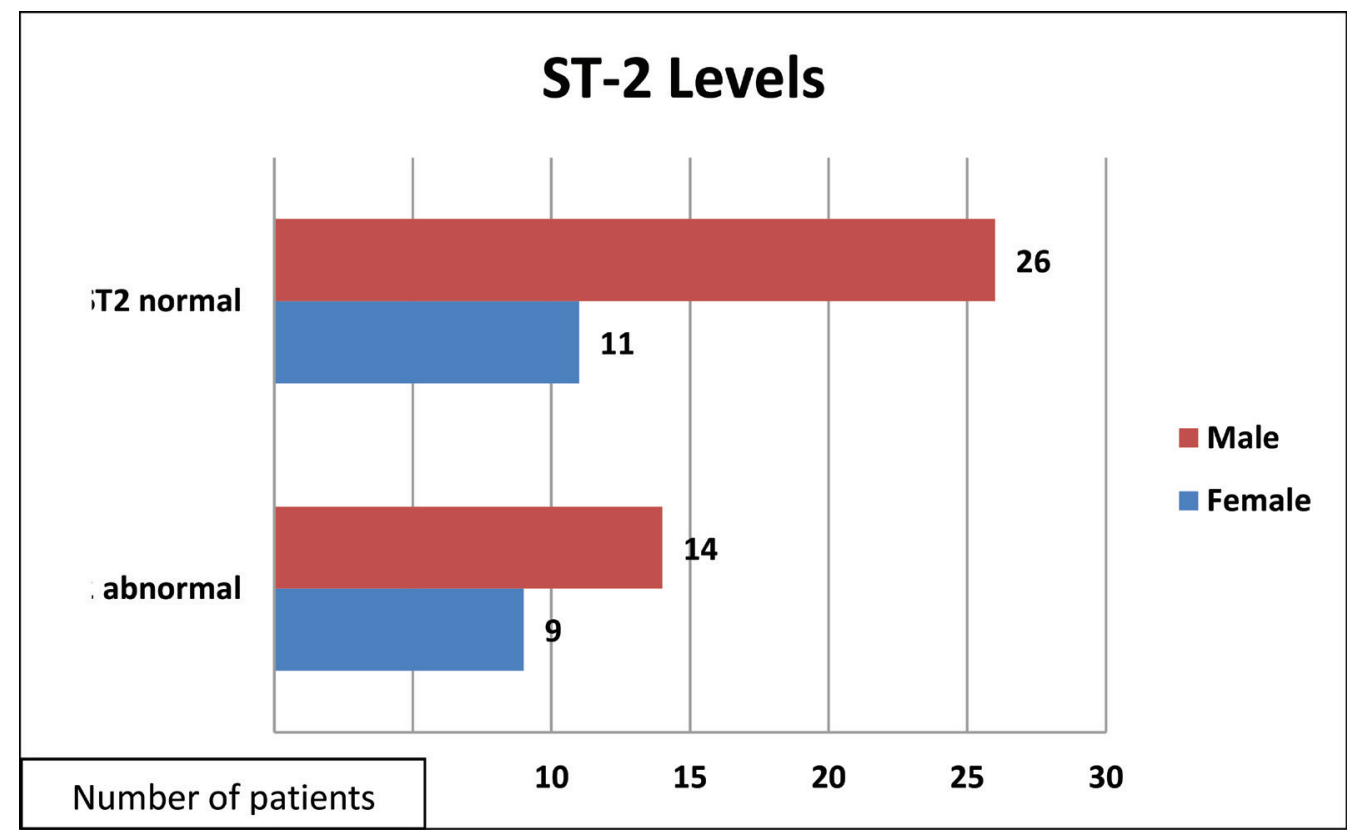

Fig. 3 ST2 levels' comparison in females versus males.

Table 1 Baseline mean ST 2 levels in females versus males

\begin{tabular}{|l|l|l|l|}
\hline $\begin{array}{l}\text { Baseline } \\
\text { biomarker level }\end{array}$ & F & M & p value* \\
\hline ST2 $(\mathrm{ng} / \mathrm{mL})$ & $61.9 \pm 71.6$ & $37.0 \pm 37.9$ & 0.159 \\
\hline
\end{tabular}

${ }^{*} p$ value is $>0.05$ (not significant).

difference did not show statistical significance. The mean ST2 levels in females were $61.9 \pm 71.6 \mathrm{ng} / \mathrm{mL}$, while in males, it was $37.0 \pm 37.9 \mathrm{ng} / \mathrm{mL}$, suggesting that ST2 levels were more elevated in females (-Table 1).
Table 2 Comparison of correlation of ST2 levels with events at the end of 1 year in males versus females

\begin{tabular}{|l|l|l|l|}
\hline At the end of 1 year & Females & Males & $p$ value* \\
\hline MACE & $9(45 \%)$ & $7(17.5 \%)$ & 0.030 \\
\hline
\end{tabular}

Abbreviations: MACE, major adverse critical events.

${ }^{*} p$ value is $<0.05$ significant.

When these risk factors were correlated with ST 2 levels, only diabetes had a significant correlation with elevated ST2 levels, resulting in a significant $p$ value and suggesting that abnormal ST2 levels are more common in diabetes patients (- Table 3 ). 
Table 3 Comparison of risk factors, echo parameters and angiographic profile with ST2 levels

\begin{tabular}{|c|c|c|c|}
\hline Variable & ST2 abnormal & ST2 normal & $p$ value \\
\hline Female & 9 & 11 & \multirow[t]{2}{*}{0.45} \\
\hline Male & 14 & 26 & \\
\hline HTN & 15 & 23 & \multirow[t]{2}{*}{0.81} \\
\hline In HTN & 8 & 14 & \\
\hline DM & 16 & 14 & \multirow[t]{2}{*}{$0.01^{*}$} \\
\hline Non-DM & 7 & 23 & \\
\hline Smoker & 10 & 18 & \multirow[t]{2}{*}{0.69} \\
\hline Nonsmoker & 13 & 19 & \\
\hline Alcoholic & 5 & 10 & \multirow[t]{2}{*}{0.64} \\
\hline Nonalcoholic & 18 & 27 & \\
\hline ACS & 15 & 25 & \multirow[t]{2}{*}{0.85} \\
\hline CSA & 8 & 12 & \\
\hline \multicolumn{4}{|c|}{ Baseline LV function: } \\
\hline Moderate & 11 & 15 & \multirow[t]{2}{*}{0.58} \\
\hline Severe & 12 & 22 & \\
\hline$E / A<1$ & 10 & 12 & \multirow[t]{2}{*}{0.38} \\
\hline$E / A>1$ & 13 & 25 & \\
\hline SVD & 11 & 21 & \multirow[t]{3}{*}{0.66} \\
\hline MVD & 9 & 10 & \\
\hline DVD & 3 & 6 & \\
\hline
\end{tabular}

Abbreviations: ACS, acute coronary syndrome; CSA, chronic stable angina; DM, diabetes mellitus; DVD, double vessel disease; HTN, hypertension; LV, left ventricular; MVD, multivessel disease; SVD, single vessel disease.

${ }^{*} p$ value is $>0.05$ (not significant).

Table 4 Echocardiographic parameters in relation to abnormal mean ST2 levels with the study population

\begin{tabular}{|l|l|l|}
\hline Variable & Mean \pm Standard deviation & $p$ value \\
\hline EDV $(\mathrm{mL})$ & $138.02 \pm 28.20$ & 0.10 \\
\hline ESV $(\mathrm{mL})$ & $94.23 \pm 22.65$ & $0.03^{*}$ \\
\hline EDD $(\mathrm{cm})$ & $5.39 \pm 0.66 \mathrm{~cm}$ & 0.07 \\
\hline ESD $(\mathrm{cm})$ & $4.27 \pm 0.71 \mathrm{~cm}$ & 0.08 \\
\hline EF $(\%)$ & $22.48 \pm 17.32$ & 0.28 \\
\hline DT $(\mathrm{m} \mathrm{sec})$ & $139.12 \pm 59.02$ & 0.05 \\
\hline E/e & $11.35 \pm 4.45$ & 0.45 \\
\hline
\end{tabular}

Abbreviations: DT, deceleration time; EDD, end diastolic diameter; EDV, end diastolic volume; EF, ejection fraction; ESD, end systolic diameter; $\mathrm{ESV}$, end systolic volume.

${ }^{*} p$ value is $>0.05$ (not significant).

Among nine female patients with abnormally elevated ST2 levels at the end of 1 year, seven patients underwent repeat revascularization, two patients had recurrent admissions for heart failure, and two patients died. There were 14 male patients with abnormally elevated ST2 levels at the end of 1 year.

Seven male patients experienced events at the end of 1 year, including four patients who had mortality, five patients who met with heart failure, and two patients who underwent repeat revascularization, and the difference is statistically significant ( $p$ value 0.03 ) (-Table 2 ). It suggested that in patients with abnormally elevated ST2 levels, females have poor prognosis than males in terms of high events including repeat revascularization and mortality with statistical significance.

In the analysis of SST2 levels of patients presented with acute dyspnoea by the PRIDE substudy, sST2 levels were found to be associated with low LV function by 2D echo high right ventricular (RV) systolic pressure and hypokinesia of RV. However, there was no association with sST2 and echocardiographic parameters in the Framingham heart study. There was a strong association of SST2 levels with diastolic dysfunction from the results of the Cardiovascular Health Study. ${ }^{18}$

In our study, when ST2 levels were correlated with echo parameters (-Tables 3 and $\mathbf{4}$ ) like EDV, ESV, EDD, ESD, EF, DT, and E/e ratio, significant correlation was seen with EF and ESV with a mean EF of $17.27 \pm 16.89 \%$ and mean ESV of $97.32 \pm 22.25 \mathrm{~mL}$ in patients with abnormally elevated ST2 levels. So, elevated ST2 levels correlate with low EF.

\section{Conclusion}

CAD patients with LV dysfunction, mean blood ST2 levels were higher in females. High ST2 levels were associated with diabetes, echocardiographic high ESV, and a low EF of LV. At the end of 1 year in patients with elevated baseline ST 2 levels, female patients had more events than males 
with a statistical significance, that is, female gender has poor prognosis compared with males if ST2 levels are elevated in patients of CAD with $\mathrm{LV}$ dysfunction.

\section{Audio File}

Audio file for this article is available at https://doi.org/10.10 55/s-0040-1708567.

\section{Conflict of Interest}

None declared.

\section{References}

1 Setoguchi S, Stevenson LW, Schneeweiss S. Repeated hospitalizations predict mortality in the community population with heart failure. Am Heart J 2007;154(2):260-266

2 Pascual-Figal DA, Januzzi JL. The biology of ST2: The international ST2 consensus panel. Am J Cardiol 2015;115(7, Suppl): 3B-7B

3 Tominaga $\mathrm{S}$. A putative protein of a growth specific cDNA from BALB/c-3T3 cells is highly similar to the extracellular portion of mouse interleukin 1 receptor. FEBS Lett 1989;258(2):301-304

4 Weinberg EO, Shimpo M, De Keulenaer GW, et al. Expression and regulation of ST2, an interleukin-1 receptor family member, in cardiomyocytes and myocardial infarction. Circulation 2002;106(23):2961-2966

5 Schmitz J, Owyang A, Oldham E, et al. IL-33, an interleukin-1-like cytokine that signals via the IL-1 receptor-related protein ST2 and induces T helper type 2-associated cytokines. Immunity 2005;23(5):479-490

6 Yancy CW, Jessup M, Bozkurt B, et al; WRITING COMMITTEE MEMBERS; American College of Cardiology Foundation/American Heart Association Task Force on Practice Guidelines. 2013 ACCF/AHA guideline for the management of heart failure: a report of the American College of Cardiology Foundation/ American Heart Association Task Force on practice guidelines. Circulation 2013;128(16):e240-e327
7 Socrates T, deFilippi C, Reichlin T, et al. Interleukin family member ST2 and mortality in acute dyspnoea. J Intern Med 2010;268(5):493-500

8 Redfield MM, Rodeheffer RJ, Jacobsen SJ. Mahoney DW, Bailey KR, Burnett JC Jr. Plasma brain natriuretic peptide concentration: impact of age and gender. J Am Coll Cardiol 2002;40(5): 976-982

9 KohliP,Bonaca MP, Kakkar R, etal.Role ofST2 in non-ST-elevation acute coronary syndrome in the MERLIN-TIMI 36 trial. Clin Chem 2012;58(1):257-266

10 Januzzi JL, Pascual-Figal D, Daniels LB. ST2 testing for chronic heart failure therapy monitoring: the International ST2 Consensus Panel. Am J Cardiol 2015;115(7(Suppl) :70B-75B

11 Tseng CCS, Huibers MM, van Kuik J, de Weger RA, Vink A, de Jonge $\mathrm{N}$. The interleukin-33/ST2 pathway is expressed in the failing human heart and associated with profibrotic remodeling of the myocardium. J Cardiovasc Transl Res 2018;11(1):15-21

12 Sabatine MS, Morrow DA, Higgins LJ, et al. Complementary roles for biomarkers of biomechanical strain ST2 and $\mathrm{N}$-terminal prohormone B-type natriuretic peptide in patients with ST-elevation myocardial infarction. Circulation 2008;117(15):1936-1944

13 Weir RA, Miller AM, Murphy GE, et al. Serum soluble ST2: a potential novel mediator in left ventricular and infarct remodeling after acute myocardial infarction. J Am Coll Cardiol 2010;55(3):243-250

14 Kunwar BK, Hooda A, Joseph G. Recent trends in reperfusion in ST elevation myocardial infarction in a South Indian tier-3 city. Indian Heart J 2012;64(4):368-373

15 Xavier D, Pais P, Devereaux PJ, et al; CREATE registry investigators. Treatment and outcomes of acute coronary syndromes in India (CREATE): a prospective analysis of registry data. Lancet 2008;371(9622):1435-1442

16 Coglianese EE, Larson MG, Vasan RS, et al. Distribution and clinical correlates of the interleukin receptor family member soluble ST2 in the Framingham Heart Study. Clin Chem 2012;58(12):1673-1681

17 Miller AM, Purves D, McConnachie A, et al. Soluble ST2 associates with diabetes but not established cardiovascular risk factors: a new inflammatory pathway of relevance to diabetes? PLoS One 2012;7(10):e47830

18 Omar F, et al. Soluble ST2 in heart failure with preserved ejection fraction. J Am Heart Assoc 2017;6:e004382 